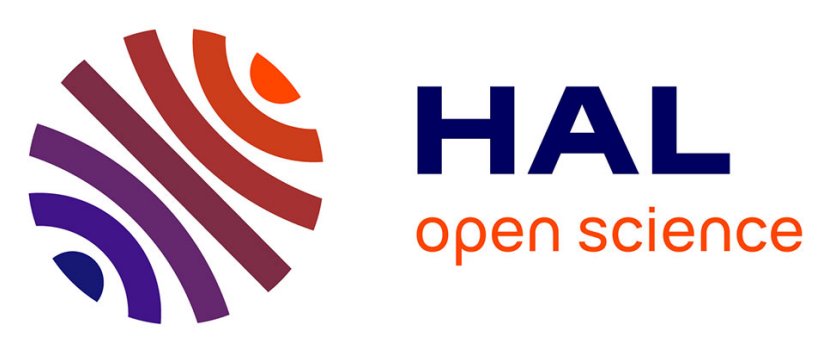

\title{
Towards real-time detection of cognitive effort in driving: contribution of cardiac measurement
}

Guillaume Pepin, Christophe Jallais, Alexandra Fort, Fabien Moreau, Jordan

Navarro, Catherine Gabaude

\section{- To cite this version:}

Guillaume Pepin, Christophe Jallais, Alexandra Fort, Fabien Moreau, Jordan Navarro, et al.. Towards real-time detection of cognitive effort in driving: contribution of cardiac measurement. Le travail humain, 2017, 80, pp.51-72. 10.3917/th.801.0051 . hal-01469240v2

\section{HAL Id: hal-01469240 \\ https://hal.science/hal-01469240v2}

Submitted on 13 Sep 2017

HAL is a multi-disciplinary open access archive for the deposit and dissemination of scientific research documents, whether they are published or not. The documents may come from teaching and research institutions in France or abroad, or from public or private research centers.
L'archive ouverte pluridisciplinaire HAL, est destinée au dépôt et à la diffusion de documents scientifiques de niveau recherche, publiés ou non, émanant des établissements d'enseignement et de recherche français ou étrangers, des laboratoires publics ou privés. 
Title: TOWARDS REAL-TIME DETECTION OF COGNITIVE EFFORT IN DRIVING: CONTRIBUTION OF CARDIAC MEASUREMENT. Short Title: TOWARDS REAL-TIME DETECTION OF COGNITIVE EFFORT IN DRIVING

Guillaume Pepin ${ }^{1}$, Christophe Jallais ${ }^{1}$, Alexandra Fort ${ }^{1}$, Fabien Moreau ${ }^{1}$, Jordan Navarro ${ }^{2}$, \& Catherine Gabaude ${ }^{1}$

${ }^{1}$ IFSTTAR-TS2-LESCOT, Bron, France

${ }^{2}$ Laboratoire d'Etude des Mécanismes Cognitifs, Université de Lyon, Bron, France

Authors:

Guillaume Pepin

IFSTTAR-TS2-LESCOT

25, Avenue François Mitterrand

69675 BRON Cedex, France

Email: guillaume.pepin@ifsttar.fr

Christophe Jallais

IFSTTAR-TS2-LESCOT

25, Avenue François Mitterrand

69675 BRON Cedex, France

Email: christophe.jallais@ifsttar.fr

Alexandra Fort

IFSTTAR-TS2-LESCOT

25, Avenue François Mitterrand

69675 BRON Cedex, France

Email: alexandra.fort@ifsttar.fr

Fabien Moreau

IFSTTAR-TS2-LESCOT

25, Avenue François Mitterrand

69675 BRON Cedex, France

Email: fabien.moreau@ifsttar.fr

Jordan Navarro

Laboratoire d'Etude des Mécanismes Cognitifs (EMC EA 3082)

Institut de Psychologie

Université Lyon 2

5, Avenue Pierre Mendès-France

69676 Bron Cedex, France

Email: jordan.navarro@univ-lyon2.fr

Catherine Gabaude

IFSTTAR-TS2-LESCOT

25, Avenue François Mitterrand

69675 BRON Cedex, France

Email: catherine.gabaude@ifsttar.fr 


\section{ABSTRACT}

In 2013, attention deficits accounted for 40 to $50 \%$ of injury accidents. Recent studies have succeeded in detecting impaired states of attention, with a view to assisting the driver, and provide a new opportunity to increase road safety. This study focuses on the detection of drivers' cognitive effort and seeks, through the study of heart rate change (HRC), to identify a sensitive indicator of cognitive effort in short time windows.

Eighteen young drivers participated in the study and took part in 8 experimental sessions where they performed a passive or active cognitive task (counting) while driving or not. The counting task had two difficulty levels (counting of beeps vs visuospatial skills and number adding). Participants' heart rates were monitored during all tasks.

Previous results recorded in laboratory conditions have been replicated during driving: during the first seconds after a cognitive effort, there is a slight deceleration and a sharp acceleration in heart rate. Conversely, in the absence of cognitive effort, simple cardiac deceleration was observed. Our study confirms that it is possible to distinguish HRC in response to a cognitive effort over short time windows by observing the grand mean of evoked cardiac responses at $0.5 \mathrm{~s}$ intervals from stimulus onset when averaged over a significant number of episodes. The new opportunities offered with this cognitive effort indicator are discussed. Recent literature data show that the removal of respiratory influence from heart rate is feasible. With such correction, it seems possible to improve the sensitivity of HRC, and HR acceleration should be observed without averaging the HRC over many trials. If this proves effective, using an algorithm to detect cognitive effort in real time, future assistance devices could warn drivers or overcome their mistakes when they no longer control driving activity because of a cognitive effort.

Keywords: Heart Rate Change, Cognitive Effort, Driving, Evoked Cardiac Response 


\section{INTRODUCTION}

Driving is a visual attentional expensive activity requiring a constant cognitive control and a constant management of the allocation of attentional resources by adopting different cognitive compromises. These compromises refer to adjustments to achieve a satisfactory, albeit not optimal, performance, while limiting the cognitive cost of the task (Hoc \& Amalberti, 2007). Attention is a multidimensional concept referring to a state in which there is an optimal level of activation to select and prioritize information in controlling the ongoing activity (Rueda, Pozuelos, \& Combita, 2015). As individual's attentional resources capacities are limited for paying attention (Wickens, 1980), performing an additional task while driving divides attentional resources so the driver can perform both tasks in parallel, which leads to deterioration in driving performance. A cognitive load is generated by the cognitive activities expected from the driver who, drawing on his/her own resources, makes a cognitive effort to perform the required mental processing (Amalberti, 2001). Recent advances in cognitive neuroscience offer new opportunities to improve our understanding of how this cognitive control operates.

In 2003, Parasuraman introduced a new interdisciplinary field called "neuroergonomics," which aspires to be the best tool for studying the "brain at work". The idea is that the interaction between ergonomics and neuroscience would improve understanding of the brain in action and that the human operator could be monitored using direct data from the neurosciences. A passive brain-computer interface would connect brain activity to a system able to provide the operator with feedback on his/her status (Roy, 2015), leading to safer interactions between the system and the operator. In practical terms, neuroergonomics can lead to the design of more efficient and safer working conditions. The potential beneficiaries include the owners of the systems in which the technologies are used. For example, a passive brain-computer interface could provide useful information to drivers, who are increasingly exposed to impaired attentional states.

In the last decade, epidemiological studies have shown that two types of impaired attentional states are contributing factors in about 40 to $50 \%$ of road accidents (Galéra, Orriols, M'Bailara, Laborey, Contrand, Ribéreau-Gayon et al., 2012; Mosedale, Purdy, \& Clarkson, 2004):

- Inattention to driving is defined as a state inducing endogenous shift of attention (Lemercier \& Cellier, 2008). The emergence of inattention can be promoted by various factors including fatigue, medication or mind wandering (MW), which corresponds to unintentional thoughts unrelated to the task (Galéra et al., 2012). MW is also defined as a shift in the contents of thought away from an ongoing task to selfgenerated thoughts and feelings (Smallwood \& Schooler, 2015). These factors will reduce the driver's ability to operate sufficient cognitive control and thus promote the emergence of inattention.

- Distraction is defined as a diversion of attention away from critical activities to competitive activities not linked with the ongoing activity; an example is cell phone use while driving (Strayer, Drews, Albert, \& Johnston, 2001). Distraction consists of 
exogenous orientation of attention to external events inside or outside the vehicle. This presents a risk for the driver since it contributes to a significant number of road accidents (Wang, Knipling, \& Goodman, 1996; Wilson \& Stimpson, 2010).

Recently, it has been shown that MW while driving is detrimental since it degrades the driver's control of the vehicle, with a decrease of speed micro-regulations and larger deviations in the vehicle's lateral position (Lemercier, Pêcher, Berthié, Valery, Vidal, \& Paubel et al., 2015). MW also increases the risk of being considered responsible for a road accident (Galéra et al., 2012). Some authors consider that this higher risk is due to a failure to scan or monitor the environment (He, Beauvale, \& Bener, 2011). Several techniques have been used to identify MW episodes using eye gaze (Uzzaman \& Joordens, 2011) and variability in lane position (Gabaude, Baracat Jallais, Bonniaud, \& Fort, 2012). Nevertheless, it seems easier to spot a distraction than inattention. Distraction can be detected when the driver looks away from the road while interacting with elements in the environment or when there is interference from a manual activity. Inattention, on the other hand, involves no easily identifiable behavioral activity. Within this framework, physiological data therefore seem to be the most able to shed light on this cognitive state.

To understand how the cognitive effort is managed by an operator, it is important to distinguish the concepts of effort and cognitive load. Cognitive load is impersonal, it corresponds to what is required by the ongoing task; effort is what the participant agrees to allocate as resources for the processing of this task, it's the investment made (Paas, 1992). In other words, the cognitive load is subjected to external constraints (dependent on the task) and effort is subjected to internal ones (dependent on the abilities, skills and motivation of the driver). So, depending on internal factors, the cognitive effort made by two people performing the same task may differ.

Studies of cognitive effort while driving could help drivers avoid exposure to cognitive overload, which is a state where the effort made exceeds the available resources required to perform the task correctly. By highlighting a real-time indicator of cognitive effort, it would seem possible to anticipate potential overload while driving. Impaired attentional states behind the wheel being ubiquitous, the indicator of cognitive effort while driving must therefore be universal. There are already several methods for recording cognitive effort in driving, especially using questionnaires as the NASA-TLX (Hart \& Staveland 1988). However, the use of introspective questionnaires does not allow real-time detection of the cognitive effort made by drivers. Moreover, questionnaires raise the problem of subjectivity and the reliance on self-report measures. These kind of introspective measures are still needed but have to be corroborated by objective and external measures such as physiological data to avoid self-report biases (Schooler \& Schreiber, 2004; Smallwood \& Schooler, 2015). Indeed in order to monitor drivers in real-time, in accordance with the neuroergonomics approach, indicators have to be objective and continuous to detect unconscious mental state such as mind-wandering. In addition, measures of lateral deviation and vehicle speed seem to be linked to the cognitive cost of a task, but are not sensitive enough to provide a rapid response regarding the presence of a cognitive effort. The indicator selected should be sufficiently 
sensitive to the presence of a cognitive effort within a strict timeframe. The purpose of this study is to highlight an indicator of cognitive effort that can be used in real time while driving. Our indicator takes into account physiological measures, particularly heart rate (HR) and its change. Previous studies have tried to highlight the driver's cognitive effort in real driving situations (Gabaude, Baracat Jallais, Bonniaud, \& Fort, 2012). Participants were asked to perform various cognitive tasks in parallel with a main driving activity. HR variability was studied conducting frequency-domain analysis over a $300 \mathrm{~s}$ time window to reach a sensitive indicator of cognitive effort. Various authors have highlighted cognitive effort indicator conducting frequency-domain analysis such as Low Frequency / High Frequency ratio of heart rate variability (Durantin, Gagnon, Tremblay \& Dehais, 2014; Hjortskov, Rissén, Blangsted, Fallentin, Lundberg \& Søgaard, 2004). Even if these studies highlighted a cognitive effort indicator, they conducted frequency-domain analysis on time window in the order of one to several minutes. Considering the complexity of the driving activity, a one minute time window is too large to detect the cognitive effort in real time. To reach this goal, the indicator has to be able to detect a cognitive effort in only few seconds. Therefore, while driving, a real-time detection of the cognitive effort cannot be done through that kind of analysis but has to be done through time-domain analysis using indicators such as the heart rate change (HRC).

The extent of cognitive effort using a HRC measurement has already been demonstrated in laboratory tasks using 6 s windows (Kaiser, Beauvale, \& Bener, 1996; Kaiser, Wronka, Barry, \& Szczudlik, 1999; Lawrence \& Barry, 2009). Various authors working on change in HR have reported differences in HRC patterns, known as response patterns, between a passive and an active cognitive task (Kaiser et al., 1996, Kaiser et al., 1999 Lawrence \& Barry, 2009). In the active task condition, participants were asked to count beeps while in the passive task they were asked to ignore the sounds heard and relax. In the passive task, simple deceleration of the HR was observed. In contrast, the active task showed a biphasic response: an early very short HR deceleration (ECR1) followed by acceleration (ECR2). In order to determine whether cardiac acceleration during cognitive processing was linked to an effort, the authors conducted a complementary study. They reproduced the same experimental conditions as previously but included patients with amyotrophic lateral sclerosis (ALS), a motor neuron disease that does not interfere with executive behavior, but impairs cognitive function. Partial losses of synaptic connections is involved, mainly in the frontal lobe. There was no difference between groups in the passive task, whereas a less pronounced, significant HR acceleration was found in the active task for ALS patients (Kaiser et al., 1999). This study corroborates the hypothesis that the ECR2 component is the manifestation of cognitive effort, this process being altered in the ALS group. In summary, cardiac deceleration in passive tasks (and slightly present in active tasks) can be associated with automatic processing of a stimulus, without any need to become involved. This is a passive and not controlled process. Conversely, the strong HR acceleration in active tasks is correlated with executive control processes involving the frontal cortex and requiring active and conscious processing (Lawrence et al., 2009). Moreover, HR acceleration seems more pronounced when the cognitive effort is greater. These results should be confirmed in order to explore the sensitivity of such an indicator because various questions are unanswered: will the ECR2 
component change with a more difficult cognitive task? Will the change be in amplitude and/or delay? Will the final cardiac acceleration be greater with a difficult cognitive task than with an easy one? Manipulation of the difficulty of the cognitive task would allow the links between cognitive effort and HRC to be specified.

According to Lawrence \& Barry (2009), these hypotheses on the recording and processing of information during active and passive cognitive tasks have been confirmed with other neurobiological correlates, and a parallel has been drawn with simultaneous brain variations. Using the same experimental protocol, the authors demonstrated strong correlations between event-related potentials (ERPs) and ECR. In this way, they concluded that the auditory N1 component (early component specific to the stimulus sensory modality) and P300 (late component nonspecific to the stimulus sensory modality) are to ERPs what the ECR1 and ECR2 components are to ECR. Another study confirmed that the slightly deceleration of HR in passive tasks is due to unintended resource allocation (Schröger, 1997; Volosin \& Horvath, 2014). This automatic allocation corroborates the hypothesis that the ECR1 component is passive and uncontrolled. Conversely, the ECR2 and P300 components reflect the cognitive effort made by the participant.

As previously mentioned, the neuroergonomics approach offers to link ergonomics and neuroscience data to improve understanding of the brain in action (Parasuraman, 2003). Cardiac markers (ECR1 and ECR2) obtained studying HRC are highly correlated with brain markers appearing simultaneously (Lawrence \& Barry, 2009). HRC seems to provide a relatively stable measure of cognitive effort through a near-instantaneous objective measurement. The present study had several objectives: first, to confirm that the use of HRC can distinguish active and passive conditions; second, HRC needs to be sensitive enough to show greater HR acceleration as task difficulty increases: when an additional cognitive task is performed, a larger HR acceleration is expected compared with conditions without an additional task. The main objective of this study, using analysis of the HRC, was to highlight a cognitive effort indicator usable while driving. Ultimately, a sensitive cardiac marker would allow an automatic driver system to monitor the real-time states of drivers (Roy, 2015).

\section{MATERIALS AND METHODS}

\section{Participants}

An online procedure was used to recruit 18 healthy volunteers from the University of Lyon (10 males, age $22.7 \pm 1.4$ ). All of them reported normal or corrected to normal visual acuity and had been holders of a valid driving license for at least 3 years. Before starting the experiment conducted at IFSTTAR Bron, participants were asked about their health status and only those without any declared cardiovascular diseases were included. All participants gave written consent and received no financial support for their participation. Throughout the experiment, participants were seated in a car wearing an ambulatory measurement system of cardiac activity. 


\section{Experimental design}

Four factors were studied: one repeated measurement (mean HRC in a $0.5 \mathrm{~s}$ window; 13 consecutive measurements recorded to analyze the shape of the response) and three withinsubject factors (cognitive activities: active vs passive; tasks: single vs dual; and difficulty levels: easy vs difficult). Each participant took part in each condition of the independent variables. In the dual task condition, while dealing with the cognitive activity, drivers also had to maintain the car speed at $70 \mathrm{Km} / \mathrm{h}$ and stay in the center of the lane.

In order to manipulate the intensity of the cognitive effort, two mental calculation cognitive tasks were implemented. In the easy cognitive task, auditory stimuli were presented and, according to the cognitive activity (active vs passive), participants had to count them or not. The protools software was used to implement beeps according to the specification provided by Kaiser et al. (1996). In the difficult cognitive task, participants memorized beforehand a grid of 25 digits or numbers (presented in 5 columns and 5 rows; in order to facilitate memorization, the units increased from one column to another and the tens from one row to the other). Direction instructions (up, down, left, right) were presented orally and the participant, in the active condition, had to move mentally within the grid and add the new number to the results previously obtained.

To ensure the successful completion of the cognitive task, in each condition, the number of stimuli varied between 7 and 13. In checking the accuracy of the answers, we checked that the cognitive task was performed correctly. All experimental conditions were partially counterbalanced. In order to avoid a learning effect (e.g., automatic counting sounds), for each task, the four passive conditions were performed before the active ones. Four groups were formed by balancing the tasks and the difficulty levels. All participants heard 77 stimuli during the experiment. In total, across all participants, 1386 events were collected in this study (Table 1).

Table 1: Number of items recorded and used in statistical analyses

\begin{tabular}{cccc}
\hline & $\begin{array}{c}\text { Number of events } \\
\text { by subject and by } \\
\text { condition }\end{array}$ & $\begin{array}{c}\text { Number of events } \\
\text { by condition }\end{array}$ & $\begin{array}{c}\text { Number of usable } \\
\text { events }\end{array}$ \\
\hline Easy/DT/Pas & 7 & 126 & 94 \\
\hline Easy/DT/Act & 12 & 216 & 212 \\
\hline Easy/ST/Pas & 13 & 234 & 215 \\
\hline Easy/ST/Act & 11 & 198 & 185 \\
\hline Diff/DT/Pas & 10 & 180 & 168 \\
\hline Diff/DT/Act & 8 & 144 & 141 \\
\hline Diff/ST/Pas & 9 & 162 & 114 \\
\hline Diff/ST/Act & 7 & 126 & 1279 \\
\hline Total & 77 & 1386 & \\
\hline Diff $=$ Difficult, DT $=$ Dual Task, ST = Single Task, Pas $=$ Passive and Act $=$ Active
\end{tabular}


Precautions were taken to allow the making of cognitive efforts even if a mistake was made in counting: a one point margin of error was allowed for the easy counting task; when using mental displacement in the grid (difficult counting task), participants had to move in the 9 central squares of the 25-square grid they had memorized; if a participant announced being outside the grid, the current trial was canceled. No participants made more than one mistake per condition. Therefore, all conditions were retained. Concerning the passive task conditions, participants were seated in the car and were asked not to pay attention to the sounds presented.

\section{Procedure}

Before the experiment started, participants were informed about the experiment and asked to give their consent. They were informed that they could stop the experiment at any time if necessary. Then, the 3 electrodes were applied and participants sat in the simulator cabin. A 5 min baseline measurement of $\mathrm{HR}$ was made for each participant prior to the experimentation. Following this baseline measurement, a short training session (2 to $5 \mathrm{~min}$ ) was performed, after which participants had to learn the calculation grid during $5 \mathrm{~min}$. Using three mental displacements in the grid, the experimenter checked that the difficult counting task was understood and feasible. When ready to perform the cognitive tasks, participants were then exposed to the 8 experimental conditions (each lasted from 5 to $9 \mathrm{~min}$ ) in a counterbalanced order. The whole experiment lasted nearly one and a half hours. After each active cognitive task, participants were asked to give the target count to the experimenter. At the end of the experiment, a debriefing session was performed.

\section{Material and apparatus}

a. Driving simulator

The driving simulator consisted of a Peugeot 308 (Peugeot S.A., Paris, France; length $=4.27 \mathrm{~m}$; width $=1.81 \mathrm{~m}$ ) surrounded by seven video projection screens (covering a total angular space of $270^{\circ}$ and vertical angular size of $47.5^{\circ}$ ). Participants drove in a straight line and curves in an urban residential zone with sparse traffic. The vehicle was equipped with a manual transmission, and the steering wheel had a force feedback system. A system of sound restitution was used for motor sounds and the instructions given to participants. A microphone, inside the car, was used to record the participants' responses.

\section{b. Heart rate measurement and pre-processing}

HR was measured using 3 electrodes via a wireless physiology solution (Biopac, MP150 using Bionomadix transmitters) in each experimental condition and during the baseline measurements. Each participant wore three electrodes connected to the Bionomadix device. The positive electrode was placed under the last left rib, the negative electrode on the manubrium of the sternum, and the ground electrode on the right side, just on top of the hip. In a test phase, it has been checked that this positioning satisfies the main conditions to collect correct cardiac signal during driving: compromising with the length of the wires and avoiding a noisy signal due to muscular activity. 
An off-line cardiotachometer method, recently published by Guerra et al (2016), was used to identify interbeat intervals (IBI) before running an extraction procedure to obtain an evoked cardiac response. The ECGLab tool box developed in MATLAB language (Azevedo de Carvalho, Da Rocha, De Oliveira Nascimento, Souza Neto, Junqueira, 2002) have been used to run an automatic detection of the R-R intervals in order to define IBI corresponding to the time between two peaks of $\mathrm{R}$ waves. At this step, the user can inspect the ECG and correct mistakes in the detection process, and also identify ectopic beats. All data sets were manually checked for outliers (1386 events) to eliminate trials (107 events) from which the cardiac signal was noisy. On average, $71( \pm 8)$ evoked cardiac responses were analyzed for each participant; half in active and half in passive conditions (see Table 1). Then, off-line transformation of the IBI into HR in beats/min was done to obtain a continuous signal. HR and IBI are defined as follows:

$$
\begin{gathered}
I B I_{n}=r_{n}-r_{n-1} \\
H R_{n}=\frac{60,000}{I B I_{n}}
\end{gathered}
$$

where $I B I_{n}$ is the interval between two peaks at a given time in milliseconds (ms), $r_{n}$ is the time of the $\mathrm{n}^{\text {th }} \mathrm{R}$ peaks and $H R_{n}$ is the heart rate in beat per minute (bpm) at the same time (Roy, 2015).

As a last step to obtain the heart rate change (HRC) every $0.5 \mathrm{~s}$, the transformation formula have been calculated as follows:

$$
H R C=H R(t)-H R\left(t_{0}\right)
$$

where $H R C$ is the change in heart rate in beat per minute (bpm), $H R(t)$ is the heart rate at any time in the time window and $H R\left(t_{0}\right)$ is the heart rate at the beginning of the time window. Indeed, each time window lasted 6 s corresponding to 13 successive HRC values including the first and the last data point. Therefore, an event-related response corresponding to HRC was obtained for each time window. It should be noted that a brief HR deceleration (ECR1) reflects stimulus recording, whereas the following HR acceleration (ECR2) reflects a cognitive load that is absent if the subject is not making a cognitive effort.

\section{Statistical analyses}

The ECR was analyzed using repeated measure ANOVA to examine response trends in the $6 \mathrm{~s}$ following stimulus onset relative to the pre-stimulus HR value (13 HRC measurements). The analysis examined cognitive activities (active vs passive), tasks (single vs dual) and difficulty levels (easy vs difficult) as within-subject factors in the design. Simple (linear, quadratic, cubic) trends over time were used to define response effects. As mentioned by Lawrence and Barry (2009), generally, a brief phasic cardiac response is indicated by a quadratic trend over a short time period and/or a cubic trend if the response is not symmetrical in the time period. This cubic trend may be supplemented/replaced by a linear trend if the response is incomplete in the time period. To specify differences between conditions, paired 
samples Student's $t$-tests were calculated comparing HRC values for each time step. These $t$ tests have not been corrected for multiple comparisons.

\section{RESULTS}

As described in Table 1, 1279 events were exploited across all conditions after removing events for which the cardiac signal was unsuitable for analysis. The results of statistical analyses are summarized in Table 2. For these analyses, all interactions between repeated measurements and our three factors of interest are presented.

Table 2: Results of statistical analyses

\begin{tabular}{c|ccc}
\hline \multicolumn{1}{c}{ Source } & Trends & $\boldsymbol{F ( 1 , 1 1 )}$ & $\boldsymbol{P}$-value \\
\hline Acti & Linear & 12.65 & $\mathrm{p}<.01^{* *}$ \\
& Quadratic & 15.77 & $\mathrm{p}<.01^{* *}$ \\
& Cubic & 2.82 & $\mathrm{p}=.12 ; \mathrm{NS}$ \\
\hline Acti*Task & Linear & 0.17 & $\mathrm{p}=.69 ; \mathrm{NS}$ \\
& Quadratic & 2.17 & $\mathrm{p}=.17 ; \mathrm{NS}$ \\
& Cubic & 3.91 & $\mathrm{p}=0.074$ \\
\hline Acti*Diff & Linear & 0.18 & $\mathrm{p}=.68 ; \mathrm{NS}$ \\
& Quadratic & 7.86 & $\mathrm{p}<.05^{*}$ \\
& Cubic & 0.08 & $\mathrm{p}=.79 ; \mathrm{NS}$ \\
\hline Acti*Diff*Task & Linear & 1.47 & $\mathrm{p}=.25 ; \mathrm{NS}$ \\
& Quadratic & 0.06 & $\mathrm{p}=.80 ; \mathrm{NS}$ \\
& Cubic & 0.41 & $\mathrm{p}=.54 ; \mathrm{NS}$ \\
\hline
\end{tabular}

Acti $=$ Activity and Diff $=$ Difficulty

1. Effect of cognitive effort on HRC

The ECRs averaged across active and passive conditions are presented in Figure 1. The impact of the activity on the response patterns gave rise to a significant linear $(F(1,11)=12.65, \mathrm{p}<.01)$ and quadratic trends $(F(1,11)=15.77, \mathrm{p}<.01)$. The response patterns followed both quadratic and cubic trends. No conclusion could be drawn from this analysis since two trends were significant. From a purely descriptive point of view, response patterns differ depending on the activity. In passive tasks, simple HR deceleration was observed while in active conditions a deceleration and an acceleration of HR were clearly visible. The two ECR components (ECR1 and ECR2) were identified. 


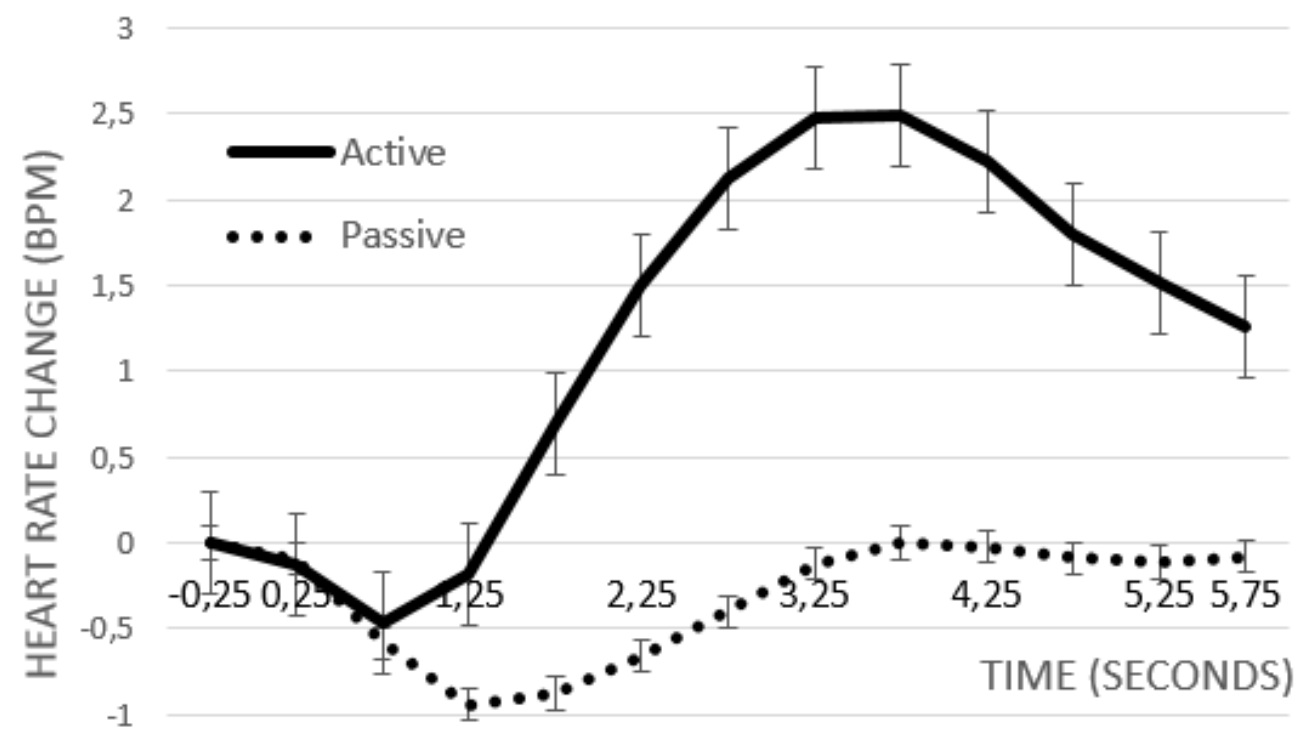

$-1,5$
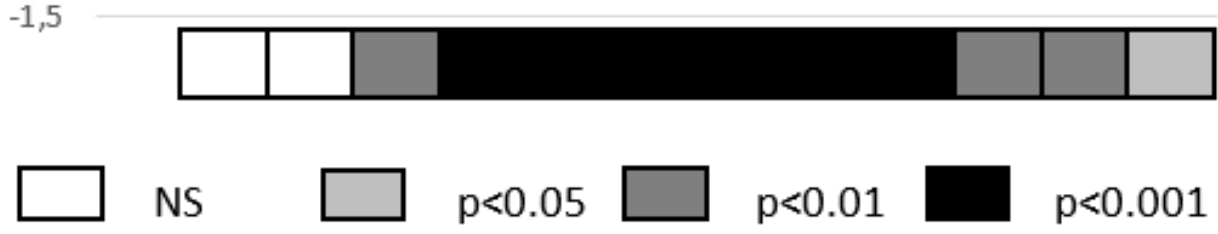

Figure 1 : Grand mean evoked cardiac responses at 0.5 s intervals from stimulus onset (obtained from raw data), ECR averaged across active vs passive conditions and statistical significance for Student's t-tests comparing mean HRC values between active and passive conditions.

To specify differences between active and passive tasks better, a paired samples Student's $t$ tests were calculated comparing HRC values between conditions for each time step. It has to be noted that all $t$-tests have not been corrected for multiple comparisons. The first significant difference occurred $1.25 \mathrm{~s}$ after stimulus onset ( $4^{\text {th }}$ data point). At this time, the HRC value for the active conditions was -0.18 and -0.94 for the passive task. The difference was statistically significant $t(17)=2.90, \mathrm{p}<.01$. For points 5 to 13 (1.75 s after stimulus onset to $5.75 \mathrm{~s}$ ), the calculated differences in HRC values between active and passive tasks were significant until the $13^{\text {th }}$ data point. $T$-values were respectively $4.81,4.70,4.72,5.14,5.56,5.31,4.10,3.09$ and 2.86 .

2. Effect of cognitive effort and additional task on HRC

A near-significant two-way interaction was found according to the activity (active vs passive) and task (simple vs dual) for the cubic trend $(F(1,11)=3.91, \mathrm{p}=.074)$ presented in Figure 2 . Interactions between activity and task for linear and quadratic trends were not significant. Even though these results are not significant, the initial HR deceleration was greater with an additional driving task during the passive task condition. In active conditions, cardiac acceleration was greater during the dual task conditions than during the simple task. 


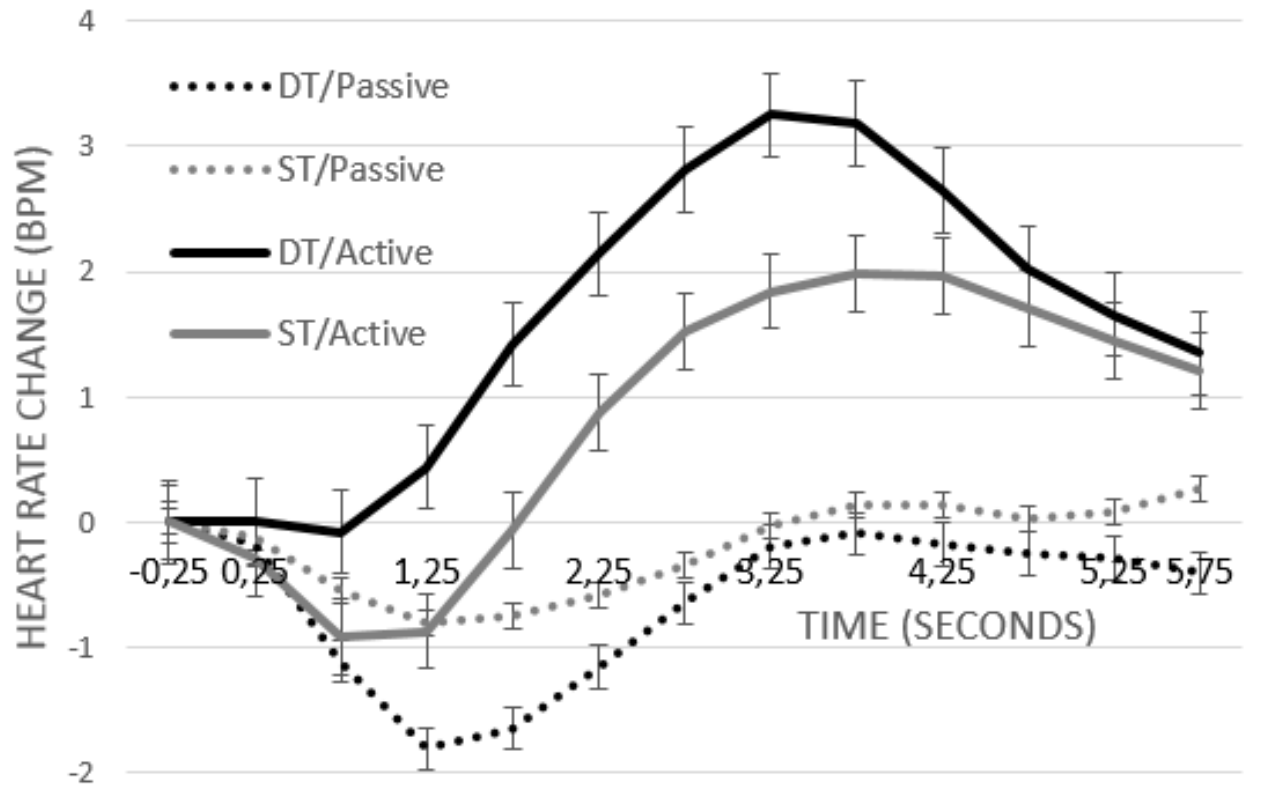

ST/Active vs ST/Passive

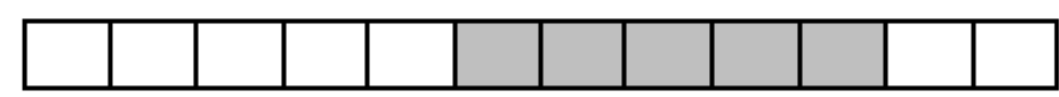

DT/Active vs DT/Passive

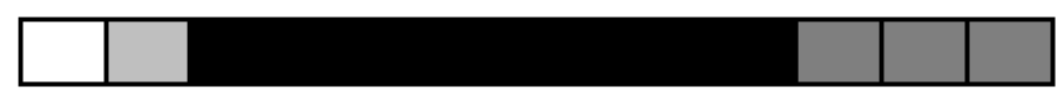

ST/Active vs DT/Active
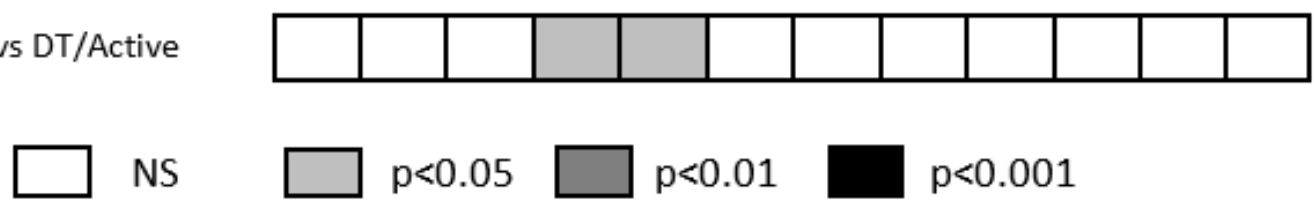

Figure 2: Grand mean evoked cardiac responses at 0.5 s intervals from stimulus onset, ECR averaged across active vs passive conditions, simple vs dual task and statistical significance for Student's t-tests comparing mean HRC values between ST/Active conditions and ST/Passive conditions, DT/Active conditions and DT/Passive conditions.

Regarding Student's $t$-tests, the first significant difference occurred between the $6^{\text {th }}$ and $7^{\text {th }}$ data point (2.75 s after stimulus onset). The HRC value for active conditions was 1.52 and 0.34 for the passive task: the difference was statistically significant $t(17)=2.14, \mathrm{p}<.05$. The differences were significant until the $11^{\text {th }}$ data point included $(4.75 \mathrm{~s})$. For points 8 to $11, t$ values were respectively 2.35, 2.67, 2.80 and 2.47 .

Considering HRC differences obtained in the dual task (with an additional driving task) for active and passive conditions, the first significant difference occurred at the $3^{\text {rd }}$ data point ( $0.75 \mathrm{~s}$ after stimulus onset). The HRC value for the dual task was -0.08 in active conditions and -1.11 in passive conditions, the difference being statistically significant $t(17)=2.46, \mathrm{p}<.05$. For points 4 to 13 (1.25 s after stimulus onset to $5.75 \mathrm{~s}$ ), the differences calculated in HRC values between active and passive tasks were all significant. The $t$-values for points 4 to 13 were respectively 4.42, 5.21, 5.14, 5.06, 4.77, 4.63, 4.56, 3.90, 3.51 and 3.43. 
3. Effect of cognitive effort and difficulty on HRC

A significant two-way interaction was found according to the activity (active vs passive) and task difficulty (easy vs difficult) for the quadratic trend $(F(1,11)=7.86, \mathrm{p}<.05)$ : Figure 3 . For linear and cubic trends, the results were not significant. These results suggest that the shape of the response is not the same according to condition and task difficulty. Thus, in passive conditions, HR returned to its baseline faster for the difficult task than for the easy one. There was even a slight HR acceleration (HRC for this task exceeded the 0 threshold in the second half of the time window). In active conditions, a larger HR initial deceleration was visible in the easy task. The HR acceleration following this deceleration was present for both easy and difficult tasks, but was greater for the difficult one.

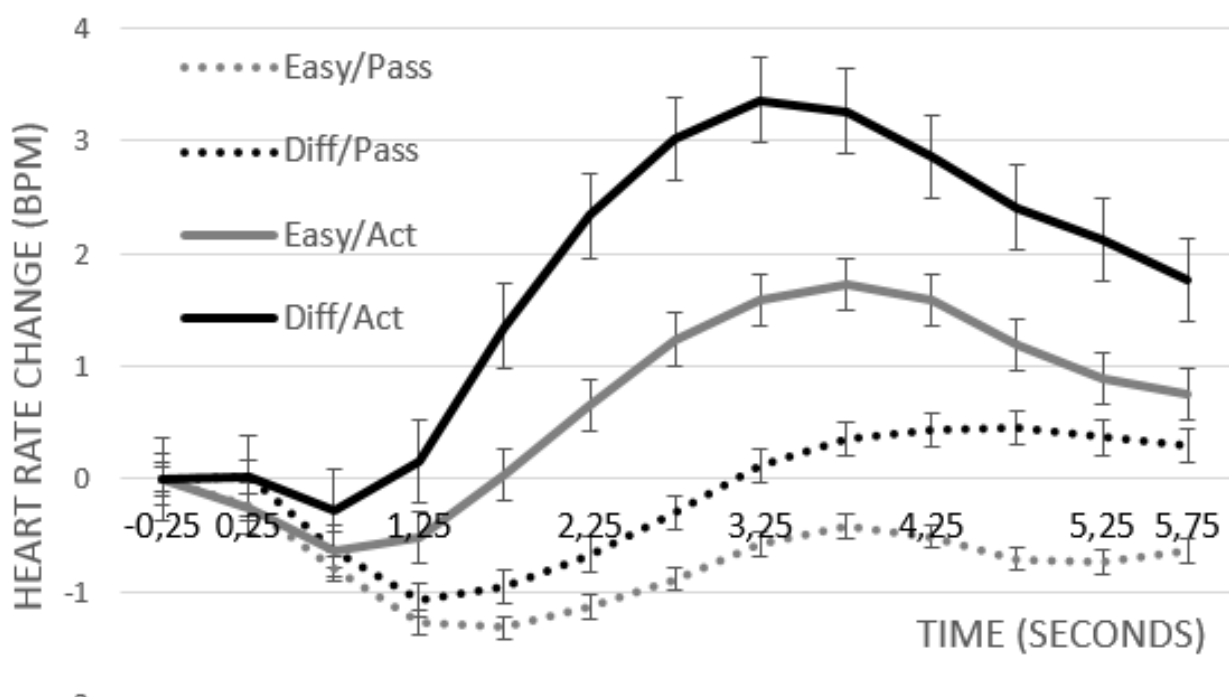

Easy/Active vs Easy/Passive

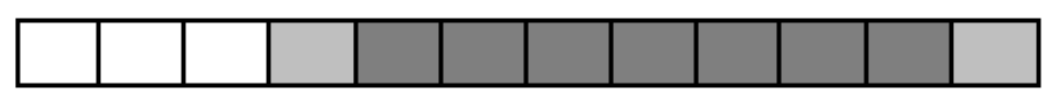

Difficult/Active vs Easy/Passive

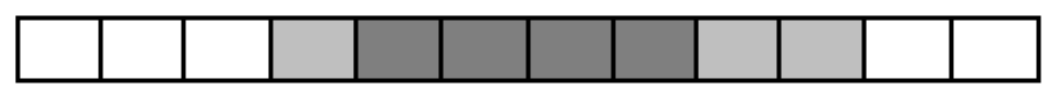

Easy/Active vs Difficult/Active
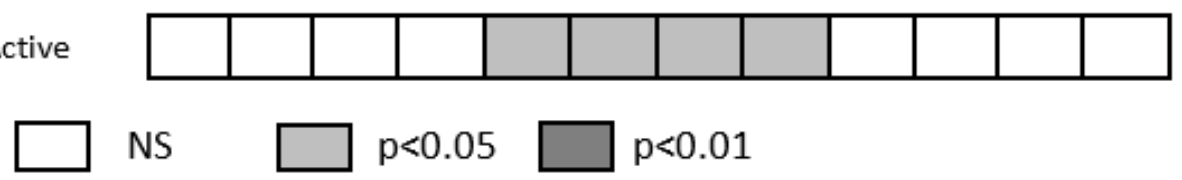

Figure 3: Grand mean evoked cardiac responses at 0.5 s intervals from stimulus onset, ECR averaged across active vs passive conditions, easy vs difficult level and statistical significance for Student's t-tests comparing HRC between easy/active conditions and easy/passive conditions, difficult/active conditions and difficult/passive conditions and easy/active conditions and difficult/active conditions

Student's $t$-tests were performed to specify differences between results obtained in the easy task for active and passive conditions. The first significant difference occurred at the $5^{\text {th }}$ data point (at $1.75 \mathrm{~s}$ after stimulus onset). HRC values during the easy task for the active conditions was -0.06 and -1.49 for the passive conditions, the difference being statistically significant $t(17)=2.66, \mathrm{p}<.05$. The differences remained significant until the $13^{\text {th }}$ data point included. Calculated $t$-values for points 6 to 13 were respectively 3.01, 3.24, 3.23, 3.34, 3.72, 3.61 and 2.99 . 
Considering HRC differences obtained in the difficult task for active and passive conditions, the first significant difference also occurred in the $5^{\text {th }}$ data point $(1.75 \mathrm{~s}$ after stimulus onset). HRC values were 1.54 for the active task and -0.94 for the passive task, the difference being statistically significant $t(17)=2.75, \mathrm{p}<.05$. For points 6 to 11 (from $2.25 \mathrm{~s}$ after stimulus onset to $4.75 \mathrm{~s}$ ), the calculated differences in HRC values for the difficult task between active and passive conditions were significant. Calculated $t$-values were respectively 3.25, 3.43, 3.45, 3.37, 2.81 and 2.26.

\section{Results by Participant}

After applying the decision criteria explained above, 9 of 18 participants (50\%) had HRC values in agreement with our assumptions for active and passive conditions. Conversely, 2 participants did not match our assumptions for active and passive tasks. The remaining 7 participants had one condition that matched our assumptions and another that did not. Among these 7 participants, 6 had passive conditions that did not match our assumptions and one who had active conditions that did not match our assumptions. Figure 4 and Figure 5 present results obtained from respectively a participant on which the indicator is sensitive enough to show ECR1 and ECR2 components and a participant on which the indicator is not sensitive enough to show these components.

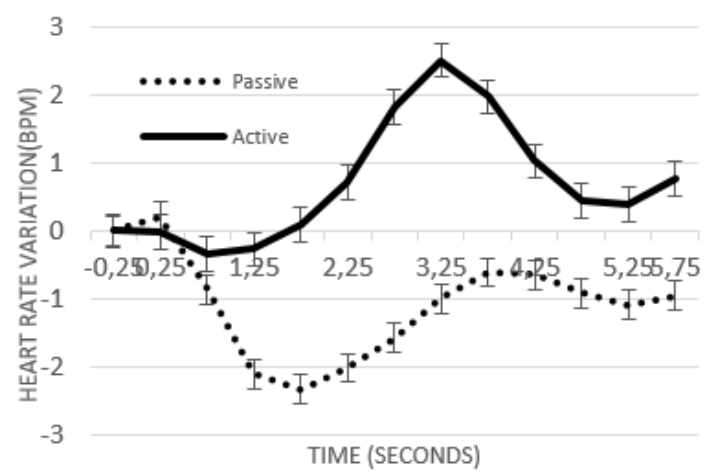

Figure 4: Example of results obtained with a participant on which the indicator is sensitive enough

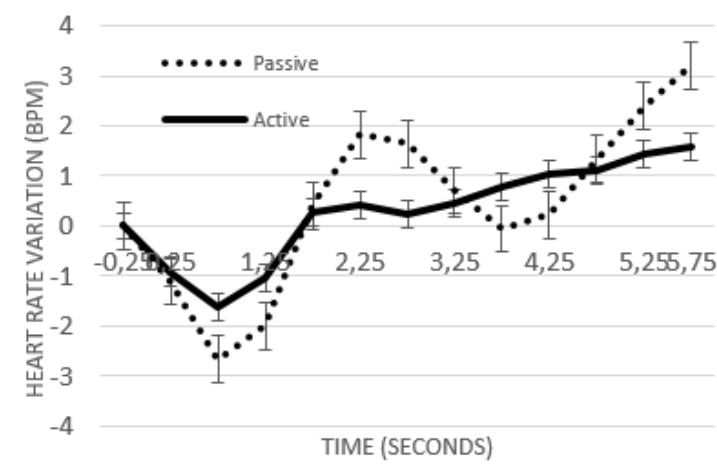

Figure 5: Example of results obtained with a participant on which the indicator is not sensitive enough

\section{DISCUSSION}

The main objective of this study was to identify, through HRC analysis during short time windows, an indicator of cognitive effort usable in driving. As a reminder, 18 participants performed cognitive tasks in 8 experimental conditions. Four factors were studied: one repeated measure (grand mean evoked cardiac response with $0.5 \mathrm{~s}$ intervals from stimulus onset) and three within-subject factors (cognitive activities: active vs passive, tasks: single vs dual and difficulty levels: easy vs difficult). The effects of cognitive activity on HRC and the interactions between the difficulty levels and the driving task on HRC are discussed before considering between-subject differences and finally the prospects for the use of such an indicator.

Cognitive activity had different effects on HRC: linear and cubic trends of the response pattern appeared significantly different between active and passive conditions. Visually, the 
13 recorded results corroborate those obtained by other authors (Kaiser et al, 1996; Kaiser et al, 1999; Lawrence et al, 2009). Results shown in Figure 1 suggest that the shape of HRC curves differs according to the nature of the cognitive activity. The biphasic response expected while performing a cognitive activity in response to auditory stimuli was highlighted. This biphasic response is composed of the ECR1 component corresponding to HR deceleration and the ECR2 component corresponding to HR acceleration. In the active conditions, a slight HR deceleration, followed by a sharp acceleration reflecting the cognitive effort was noticeable. Supplementary analyses were conducted to describe differences in HRC pattern. Student's $t$ tests were calculated at each time step, and differences between the two activity conditions were revealed from the fourth data point. This cognitive effort indicator has been sensitive enough to rapidly distinguish effort conditions from rest conditions (from $1.25 \mathrm{~s}$ after stimulus onset). Thus, it has been possible to highlight HR acceleration reflecting the driver's cognitive effort using HRC over short time windows.

The interaction between activity and the task's difficulty highlighted the impact of the cognitive task difficulty level on the shape of HRC. The interaction showed that the quadratic trend pattern appeared significant in the analysis of variance. The shape of HRC curves differed according to activity and task difficulty levels. In the active task conditions, the amplitude of the ECR2 component of HRC curve was larger for the difficult task than for the easy one. This result confirms that the two tasks used in this study have generated two different cognitive efforts. Counting sounds required a moderate cognitive effort while the second task (which was more complex and involved mental displacement, memory recall and mental calculation) required greater cognitive effort. Moreover, to our knowledge, no study had investigated the effect of the lexical processing of words on HRC. Thus, it is possible that these stimuli had a different impact on cognitive effort than beeps. Indeed, words are more complex stimuli than simple beeps and lexical treatment required could change the depth of processing of these stimuli. Just hearing words may lead to additional cognitive processing related to the extraction of their meaning. Yet the return of HRC to its baseline in the passive conditions was faster for the difficult task than for the easy task, but HRC also went above the baseline (Figure 3). Thus, increasing the task difficulty level leaded to changes in the shapes of HRC: the ECR2 component was more pronounced between $1.75 \mathrm{~s}$ and $3.25 \mathrm{~s}$ after stimulus onset, when the difficulty level increases. The impact of the types of stimuli on HRC is not clearly established in the literature and these possible variations will have to be taken into account in future studies. Further studies should explore the impact of lexical processing on the ECR1 and ECR2 components and thus sharpen understanding of the links between HRC and cognitive effort. Such a study could help understanding why the return to the baseline was faster in passive conditions for the difficult task than for the easy one. Two types of stimuli were used in this study and in both cases HRC was sensitive enough to distinguish active and passive conditions. With such an indicator (obtained in a very short period of time), if the driving situation requires a supplementary cognitive effort, we can imagine that cognitive overload will be detected and so can be managed.

The activity (active vs passive) and task (single vs dual) interactions allowed us to study the feasibility of the driver's cognitive effort assessment even during driving. Statistical analyses 
showed a significant cubic trend in the HRC pattern. By comparing curves obtained according to activity and task, there was a larger recorded HR acceleration while performing a dual task than a single task. There was an additive effect of the driving task: the cognitive effort was greater when an added task (e.g., driving) was required simultaneously, which is consistent with our hypothesis. From a purely descriptive standpoint, the shape of HRC differed between active and passive conditions, whether with or without additional driving activity. The ECR2 component was more pronounced in active task conditions, while the ECR1 component was more pronounced in passive task conditions. Calculated $t$-values in active and passive conditions for the simple task showed a significant difference from $2.75 \mathrm{~s}$ after stimulus onset. This means that without any additional driving task, it was possible to highlight the difference between active and passive conditions in less than three seconds after the beginning of the cognitive effort. Focusing on HRC differences obtained while performing an additional driving activity for active and passive conditions, the first significant difference occurred at the $3^{\text {rd }}$ data point ( $0.75 \mathrm{~s}$ after the stimulus onset) and persisted until the end of the time window. Thus, despite a low number of participants (e.g., 18), it is possible to differentiate active from passive conditions while driving in less than a second after the stimulus onset.

In active conditions, the extent of HR acceleration was greater with an additional driving task than without, although this effect was not significant (Figure 2). It is therefore likely that the intensity of HR acceleration in active conditions reflected the "amount" of effort made by the participant. This leads to the assumption that the greater the effort made by the participant to solve the task the more the HR accelerates. Another result seemed to corroborate this assumption: the ECR2 component for the difficult task was more pronounced (sharper HR acceleration) than the ECR2 component for the easy task (Figure 3). Accordingly, $t$-tests shown statistical differences between an active easy task and an active difficult one between 1.75 and $3.25 \mathrm{~s}$ after the stimulus onset. On the only basis of HRC, the effect of the task difficulty can be seen less than 2 seconds after the beginning of the tasks. Thus it seems possible that the heart rate change might be a quantitative indicator of the driver's cognitive effort and could provide information on the amount of effort produced. Nevertheless, effort reflects not only the participant's motivation but also his or her capacities. A person who has strong mental calculation abilities should, logically, furnish a smaller cognitive effort in solving tasks. To test this assumption on the proportionality between effort and HR acceleration, in future studies, a mental calculation test associated with a subjective evaluation of task difficulty collected through the NASA-TLX questionnaire (Hart \& Staveland, 1988) should be proposed to each participant to control for these variables.

Averaging HRC by participant highlighted substantial differences between individuals in evoked cardiac responses. These differences might be due to the impact of breathing, disparity in attention resources or disparity in motivation and personal goals. Indeed, heart rate and breathing have close links that should be taken into account ${ }^{1}$ (Choi \& Guttierrez-Osuna,

\footnotetext{
${ }^{1}$ Each breath slightly changes HR: inspiration accelerates it and expiration decreases it. This phenomenon is called respiratory sinus arrhythmia. The temporary lifting of parasympathetic tone during inspiration explains this HR acceleration and therefore the shortening of R-R intervals. Thus, respiratory data have to be taken into account in order to use online correction of HR.
} 
2011). In this first study conducted in a driving context without recording breathing, it was impossible to understand the respective influence of these two factors. It is accepted that breathing impacts HR (Marieb \& Hoehn, 2014), but there are also individual differences in attention capacities (Rueda et al., 2015). The allocation of attentional resources and attentional capacities seem to be linked to some motivational processes according to the task demands: the higher the task demand the higher the motivation (Braver, 2015). In the same vein, Young and Stanton (2002) showed that a reduction of mental workload while using automation in driving could have shrunk the ability to devote appropriate levels of attention to a secondary task. Other internal factors such as emotions can have some effects on the allocation of attention. For example, Techer et al. (2015) demonstrated that anger could increase the alerting network, while happiness could impair the executive control (Mitchell \& Phillips, 2007). Thus, it could be useful, for further studies, to take into account questionnaires that assess personal factors such as motivation, emotions so as to enlighten results obtained by physiological data, cardiac response being supplemented by galvanic skin response for example.

If the evoked cardiac response is influenced by cognitive abilities then these individual differences could impact HRC and therefore the participant's response patterns. How can we be sure that these different response patterns will not hinder the detection of cognitive effort? By studying the results per participant according to the activity (active vs passive), eleven of the thirty-six experimental conditions ${ }^{2}(30.6 \%)$ showed HRC patterns consistent with our assumptions. But with the data collected in the present study, it was impossible to understand if these variations were due to breathing or disparity in attentional resources. So, in a future study, it would be interesting to compare the individual differences in response patterns according to activity (active vs passive) before and after the removal of the influence of breathing on HR. This can be realized using a cardiorespiratory model for paced breathing (Choi et al., 2011). We hypothesize that the influence of breathing is greater than the influence of individual differences in attention resources. Thus, in our opinion, removing the influence of breathing on HR would drastically reduce individual variability, and so our cognitive effort indicator would become more sensitive. Nevertheless, the question regarding the impact of resources allocation still remains.

Finally, it appears that measurement of cognitive effort was possible by analyzing HRC over short time windows averaged for a large number of events. About $70 \%$ of active and passive conditions obtained after averaging per participant were consistent with the assumptions made. In the literature, it is accepted that HR and breathing are closely linked (Choi et al, 2011; Marieb et al, 2014). In future studies, to define a method to detect the driver's cognitive effort in real time it will be necessary to take into consideration the respiratory data. In order to subsequently perform an HR correction using these respiratory data, inspiratory and expiratory volumes can be recorded using the method suggested by Choi \& Gutierrez-Osuna (2011). In our future works, a correction algorithm (an autoregressive moving average with an

\footnotetext{
218 participants performed cognitive tasks in 8 experimental conditions. The HRC values were averaged for active and passive conditions. This led to 36 trials.
} 
exogenous inputs model) will be used to remove the respiratory influence from the cardiac data and improve the sensitivity of the HRC indicator.

With the gradual automation of vehicles, a device to monitor attentional states could eventually pinpoint times when the driver's cognitive effort increases. In the light of the number of victims each year of road crashes caused by driver inattention, cognitive load management could be a sustainable solution. The cognitive effort indicator highlighted here constitutes the foundations of such a solution.

\section{Acknowledgments}

Special thanks to Daniel Ndiaye and LEPSIS for their help in the preparation of the simulated driving scenarios.

\section{Références}

Amalberti, R. (2001). La Conduite de systèmes à risques, 2e édition. Paris: Presses Universitaires de France - PUF.

Azevedo de Carvalho, J.L., Ferreira da Rocha, A., Assis de Oliveira Nascimento, F., Souza Neto, J., \& Junqueira Jr., L.F. (2002). Development of a MatLab Software for Analysis of Heart Rate Variability. In: 6th International Conference on Signal Processing Proceedings, 1488-1491.

Bornstein, M., \& Bradley, R. (2003). Socioeconomic Status, Parenting, and Child Development. New York: Routledge.

Braver, T. (2015). Motivation and cognitive control. Psychology Press: New York, NY.

Choi, J., \& Gutierrez-Osuna, R. (2011). Removal of respiratory influences from heart rate variability in stress monitoring. IEEE Sensors Journal, 11(11), 2649-2656.

Gabaude C., Baracat B., Jallais C., Bonniaud M., Fort A. (2012). Cognitive load measurement while driving. In D. de Waard, K. Brookhuis, F. Dehais, C. Weikert, S. Röttger, D. Manzey, S. Biede, F. Reuzeau, \& P. Terrier (Eds.) (2012). Human Factors: a view from an integrative perspective. Proceedings HFES Europe Chapter Conference Toulouse, 6780. ISBN 978-0-945289-44-9. Available from http://hfes-europe.org.

Galéra, C., Orriols, L., M'Bailara, K., Laborey, M., Contrand, B., Ribéreau-Gayon, R., Masson, F., Bakiri, S., Gabaude, C., Maury, B., Lemercier, C., Cour, M., Bouvard, M., \& Lagarde, E. (2012). Mind wandering and driving: responsibility case-control study. BMJ, 345(dec13 8), e8105-e8105.

Guerra, P. M., Sánchez-Adam, A., Miccoli, L., Polich, J., \& Vila, J. (2016). Heart rate and P300: Integrating peripheral and central indices of cognitive processing. International Journal of Psychophysiology: Official Journal of the International Organization of Psychophysiology, 100, 1-11. 
Hart, S. G., \& Steveland, L. E. (1988). Development of NASA-TLX (Task Load Index): Results of empirical and theoretical research. In P. A. Hancock \& N. Meshkati (Eds.) Human mental Workload. Amsterdam: North Holland Press.

He, J., Becic, E., Lee, Y., \& McCarley, J. (2011). Mind wandering behind the wheel: performance and oculomotor correlates. Human Factors, 53(1), 13-21.

Hoc, J.M., \& Amalberti, R. (2007). Cognitive Control Dynamics for Reaching a Satisficing Performance in Complex Dynamic Situations. Journal of Cognitive Engineering and Decision Making, 1, 22-55.

Kahneman, D. (1973). Attention and effort. Englewood Cliffs, NJ: Prentice-Hall.

Kaiser, J., Beauvale, A., \& Bener, J. (1996). The evoked cardiac response as a function of cognitive load in subjects differing on the individual difference variable of reaction time. Acta Neurobiologiae Experimentalis, 56(1), 209-213.

Kaiser, J., Wronka, E., Barry, R. J., \& Szczudlik, A. (1999). Evoked cardiac response components in cognitive processing: differential effects of amyotrophic lateral sclerosis. Acta Neurobiologicae Experimentalis, 59(4), 329-334.

Lawrence, C. A., \& Barry, R. J. (2009). ERPs and the evoked cardiac response to auditory stimuli: Intensity and cognitive load effects. Acta Neurobiologicae Experimentalis, 69(4), 552-559.

Lemercier, C., \& Cellier, J.-M. (2008). Les défauts de l'attention en conduite automobile: inattention, distraction et interférence. Le travail humain, 71(3), 271-296.

Lemercier, C., Pêcher, C., Berthié, G., Valéry, B., Vidal, V., Paubel, P.-V., Cours, M., Fort, A., Galéra, C., Gabaude, C., Lagarde, E., Maury, B., 2014. Inattention behind the wheel: how factual internal thoughts impact attentional control on driving. Safety Science, 62, 279-285.

Lundwall R. A., Guo D. C., Dannemiller J. L. (2012). Exogenous visual orienting is associated with specific neurotransmitter genetic markers: A population-based genetic association study. PLoS One 7(2).

Marieb, Elaine., Hoehn, Katja (2014). Anatomie et physiologie humaines. Adaptation de la 9e édition américaine.

Mitchell, R.L.C., \& Phillips, L.H. (2007). The psychological, neurochemical and functional neuroanatomical mediators of the effects of positive and negative mood on executive functions. Neuropsychologica, 45, 617-629.

Mosedale, J., Purdy, A., \& Clarkson, E. (2004). Contributory factors to road accidents. Transport Statistics: Road Safety. London: Department for Transport. 
Paas, F. G. (1992). Training strategies for attaining transfer of problem-solving skill in statistics: A cognitive-load approach. Journal of Educational Psychology, 84(4), 429-434.

Parasuraman, R. (2003). Neuroergonomics: Research and practice. Theoretical Issues in Ergonomic Science, 4(1-2), 5-20

Rosario Rueda, M., P. Pozuelos, J., M. Cómbita, L. (2015). Cognitive Neuroscience of Attention From brain mechanisms to individual differences in efficiency. AIMS Neuroscience, 2(4), 183-202.

Roy, R.N. (2015). Etude des corrélats électrophysiologiques pour la discrimination d'états de fatigue et de charge mentale : apports pour les interfaces cerveau-machine passives. Thèse de Doctorat, Université de Grenoble, Alpes, France.

Schröger, E. (1997). On the detection of auditory deviations: A pre-attentive activation model. Psychophysiology, 34(3), 245-257. http://doi.org/10.1111/j.1469-8986.1997.tb02395.x

Smallwood, J., \& Schooler, J. W. (2015). The science of mind wandering: empirically navigating the stream of consciousness. Annual review of psychology, 66, 487-518.

Strayer, D. L., Drews, F. A., Albert, R. W., \& Johnston, W. A. (2001). Cell phone induced perceptual impairments during simulated driving. In D. V. McGehee, J. D. Lee, \& M. Rizzo (Eds.).

Techer, F., Jallais, C., Fort, A., \& Corson, Y. (2015). Assessing the impact of anger state on the three attentional networks with the ANT-I. Emotion, 15(3), 276-280

Uzzaman, S., \& Joordens, S. (2011). The eyes know what you are thinking: eye movements as an objective measure of mind wandering. Consciousness and Cognition, 20(4), 18821886.

Volosin, M., \& Horváth, J. (2014). Knowledge of sequence structure prevents auditory distraction: An ERP study. International Journal of Psychophysiology, 92(3), 93-98.

Wang, J.-S., Knipling, R. R., \& Goodman, M. J. (1996). The role of driver inattention in crashes: New statistics from the 1995 Crashworthiness Data System. In 40th Annual proceedings of the association for the advancement of automotive medicine (p. 377392).

Wilson, F. A., \& Stimpson, J. P. (2010). Trends in fatalities from distracted driving in the United States, 1999 to 2008. American journal of public health, 100(11), 2213-2219.

Young, M. S. \& Stanton, N. A. (2002). Malleable Attentional Resources Theory: A new explanation for the effects of mental underload on performance. Human Factors, 44(3), 365-375 